\title{
Numerical solutions of nonlinear fractional differential equations by variational iteration method
}

\author{
A. S. Nagdy*, KH. M. Hashem \\ Mathematics Department, Faculty of Science, Zagazig University, Zagazig, Egypt.
}

\begin{abstract}
In this paper, numerical techniques are used for solving boundary value problems of nonlinear fractional differential equations. Variational iteration method is applied to approximate solutions for this equation with boundary conditions. Numerical examples are presented to illustrate the efficiency and accuracy of the proposed method, and we compare between the numerical solutions and the exact solution of these examples.
\end{abstract}

Keywords: Variational iteration method, Caputo fractional derivative, nonlinear of fractional differential equations.

2020 MSC: 6SD30, 65R20, 45F05, 26A33.

(C)2021 All rights reserved.

\section{Introduction}

In recent years, many researchers have been focused on the numerical solutions of fractional differential equations. They are generalizations of differential equations that replace integral order derivatives by fractional order derivatives $[11,25]$. In general, differential equations are applied on describing dynamic phenomena in different fields such as physics, biology and chemistry. However, for some complicated systems the common simple differential equations cannot provide agreeable results. Therefore in order to obtain better models, fractional differential equations are employed instead of integer order one $[2,5,6]$. On the other hand the exact solutions for the majority of nonlinear of fractional differential equations are not known. Then numerical techniques and approximates are used such as polynomials method [21], wavelet method [3, 26], B-spline operational matrix method [9], variational iteration method, [8, 10, 17, 22], Adomian decomposition, method [15, 16], the collocation method [19], and fractional differential transform method [1].

In this article, we consider the nonlinear of fractional differential equations of the form

$$
D_{*}^{\alpha} y(t)+g(t)[y(t)]^{m}=f(t), \quad 0 \leqslant t \leqslant 1, \quad 1<\alpha \leqslant 2,
$$

subject to the boundary conditions

$$
y(0)=\rho,
$$

\footnotetext{
*Corresponding author

Email addresses: abbas775@hotmail.com (A. S. Nagdy), khaledmath86@gmail .com (KH. M. Hashem)

doi: $10.22436 /$ jnsa.014.02.01
}

Received: 2020-03-04 Revised: 2020-03-29 Accepted: 2020-04-08 


$$
y(1)=\sigma,
$$

where $\rho, \sigma$ are constants, $y(t)$ is the solution of $(1.1)$ in $[0,1], f(t), g(t)$ are a known functions, $m$ is a positive integer. This problem is studied in [14] when $g(t)=1$ by alternative Legendre polynomials method when $0<\alpha \leqslant 1$. In this paper we used variational iteration method for solving this problem. This paper is organized as follows. In Section 2, we present some definitions and notations. Section 3 , is devoted to the construct our numerical solutions for fractional differential equations by variational iteration method. Finally, in Section 4, we present some examples to show the efficiency and accuracy of the method and compared with their results by their exact solutions.

\section{Some definitions and notations}

In this section we present some basic definitions and properties of the fractional calculus theory, which are used in this paper.

Definition $2.1([4])$. A real function $f(t), t>0$, is said to be in the space $C_{\alpha}, \alpha \in R$, if there exists a real number $p>\alpha$, such that $f(t)=t^{p} f \leqslant(t)$, where $f \leqslant(t) \in C[0, \leqslant)$.

Definition 2.2 ([18]). A real function $f(t), t>0$, is said to be in the space $C_{\alpha}^{k}, k \in N$, if $f^{k} \in C_{\alpha}$.

Definition 2.3 ([4]). $\mathrm{I}^{\alpha}$ denotes the fractional integral operator of order $\alpha$ in the sense of RiemannLiouville, defined by:

$$
I^{\alpha} f(t)= \begin{cases}\frac{1}{\Gamma(\alpha)} \int_{0}^{t} \frac{f(x)}{(t-x)^{1-\alpha}} d x, & \alpha>0, \\ f(t), & \alpha=0 .\end{cases}
$$

Definition 2.4 ([18]). Let $f \in C_{-1}^{m}, m \in N$. Then the Caputo fractional derivative off $(t)$, is defined by:

$$
D_{*}^{\alpha} f(t)= \begin{cases}\frac{1}{\Gamma(m-1)} \int_{0}^{x} \frac{f^{(m)}(x)}{(t-x)^{\alpha-m+1}} d x, & 0 \leqslant m-1<\alpha \leqslant m, \\ \frac{d^{m} f(t)}{d x^{m}}, & \alpha=m \in N .\end{cases}
$$

Now, we introduce some basic properties of fractional operator listed as below [12]:

For $f \in C_{\mu}, \mu \leqslant-1, \gamma \leqslant-1, \alpha, \beta \leqslant 0$ :

(i) $I^{\alpha} I^{\beta} f(t)=I^{\alpha+\beta} f(t)=I^{\beta} I^{\alpha} f(t)$;

(ii) $\mathrm{I}^{\alpha} \mathrm{t}^{\gamma}=\frac{\Gamma(\gamma+1)}{\Gamma(\alpha+\gamma+1)} \mathrm{t}^{\alpha+\gamma}$;

(iii) $D_{*}^{\alpha}\left[I^{\alpha} f(t)\right]=f(t)$;

(iv) $I^{\alpha}\left[D_{*}^{\alpha} f(t)\right]=f(t)-\sum_{k=0}^{m-1} f^{k}\left(0^{+}\right) \frac{t^{k}}{k !}, 0 \leqslant m-1<\alpha \leqslant m \in N$.

\section{Analysis of the numerical method}

Consider the nonlinear fractional differential equation (1.1) with initial conditions (1.2) and (1.3). According to variational iteration method (VIM) $[8,10,17,22]$, we construct the correction functional for (1.1) as:

$$
y_{i+1}(t)=y_{i}(t)+I^{\beta}\left[\mu\left(D_{*}^{\alpha} y_{i}(t)+g(t)\left[\widetilde{y_{i}}(t)\right]^{m}-f(t)\right)\right],
$$

or

$$
y_{i+1}(t)=y_{i}(t)+\int_{0}^{t}\left(\frac{(t-s)^{\beta-1}}{\Gamma(\beta)} \mu(s)\right)\left(D_{*}^{\alpha} y_{i}(t)+g(t)\left[\widetilde{y}_{i}(t)\right]^{m}-f(t)\right) d s,
$$


where $I^{\beta}$ is the Riemann-Liouville fractional integral operator of order $\beta=\alpha+1-m, m \in N, \mu$ is a general Lagrange multiplier and $\widetilde{y}$ denotes restricted variation, i.e., $\delta \widetilde{y}_{k}=0$. We make some approximation for the identification of an approximate Lagrange multiplier, so the correctional functional (3.2) can be approximately expressed as:

$$
y_{i+1}(t)=y_{i}(t)+\int_{0}^{t} \mu(s)\left(\left(D_{*}^{2} y_{i}(t)+g(t)\left[\tilde{y}_{i}(t)\right]^{m}-f(t)\right)\right) d s .
$$

Making the above correction functional stationary, we have the following stationary conditions:

$$
1-\left.\mu^{\prime}(s)\right|_{t=s}=0,\left.\quad \mu(s)\right|_{t=s}=0 .
$$

This gives the following Lagrange multiplier

$$
\mu(s)=s-t .
$$

We obtain the following iteration formula by substitution of (3.3) into functional (3.1)

$$
\left.y_{i+1}(t)=y_{i}(t)-(\alpha-1)\right) I^{\alpha}\left[\left(D_{*}^{\alpha} y_{i}(t)+g(t)\left[y_{i}(t)\right]^{m}-f(t)\right)\right] .
$$

The initial approximation $y_{0}(t)$ can be chosen in a form which satisfies the boundary condition (1.2), hence $y_{0}(t)=\rho+A t$, where $y^{\prime}(0)=A$.

According to variational iteration method and by using the equation (3.4); an iteration scheme for the equation (3.4) can be constructed as follows:

$$
y_{0}(t)=\rho+A t, \quad y_{i+1}(t)=y_{i}(t)-(\alpha-1) I^{\alpha}\left[\left(g(t)\left[y_{i}(t)\right]^{m}-f(t)\right)\right] .
$$

\section{Examples}

In this section, we apply variational iteration method to solve four examples of nonlinear fractional differential equations, compared with their results by their exact solutions.

Example 4.1. Consider the following nonlinear fractional differential equation

$$
D_{*}^{\alpha} y(t)+t y^{2}(t)=t^{3}, \quad 1<\alpha \leqslant 2,
$$

subject to the boundary conditions

$$
y(0)=0, \quad y(1)=1,
$$

where the exact solution at $\alpha=2$ is

$$
y(t)=t .
$$

The initial approximation $y_{0}(t)$ can be chosen by a form which satisfies the condition $y(0)=0$, hence $y_{0}(t)=A t$. Substituting the initial condition (4.2) into equation (3.5), we obtain the following recursive relations:

$$
y_{0}(t)=A t, \quad y_{i+1}(t)=y_{i}(t)-(\alpha-1) I^{\alpha}\left[t y_{i}^{2}(t)-t^{3}\right]
$$

hence, we have

$$
\begin{aligned}
& y_{0}(t)=A t, \\
& y_{1}(t)=y_{0}(t)-(\alpha-1) I^{\alpha}\left[t y_{0}{ }^{2}(t)-t^{3}\right], \\
& y_{2}(t)=y_{1}(t)-(\alpha-1) I^{\alpha}\left[t y_{1}{ }^{2}(t)-t^{3}\right], \\
& y_{3}(t)=y_{2}(t)-(\alpha-1) I^{\alpha}\left[t y_{2}{ }^{2}(t)-t^{3}\right] .
\end{aligned}
$$


By using the above recursive relationship, we have

$$
y_{0}(t)=A t, \quad y_{1}(t)=A t-(\alpha-1)\left(\frac{\Gamma(4)}{\Gamma(\alpha+4)}\left(A^{2}-1\right) t^{\alpha+3}\right),
$$

where $A$ can be determined by using the boundary condition $y(1)=1$ in $y_{1}(t)$ (see Table 1 ).

Table 1: Values of the constant $A$ for different value of $\alpha$.

\begin{tabular}{|c|c|c|c|c|}
\hline & $\alpha=1.25$ & $\alpha=1.5$ & $\alpha=1.75$ & $\alpha=2$ \\
\hline $\mathrm{A}$ & 1 & 1 & 1 & 1 \\
\hline
\end{tabular}

The numerical solutions obtained for the equation (4.1) by using VIM are the same exact as solution $y(t)=t$ when $\alpha=1.25,1.5,1.75$, and $\alpha=2$.

Example 4.2. Consider the following nonlinear fractional differential equation

$$
D_{*}^{\alpha} y(t)+t y^{2}(t)=2+t^{5}, \quad 1<\alpha \leqslant 2,
$$

subject to the boundary conditions

$$
y(0)=0, \quad y(1)=1
$$

where the exact solution at $\alpha=2$ is:

$$
y(t)=t^{2}
$$

The initial approximation $y_{0}(t)$ can be chosen in the form $y_{0}(t)=A t$. Substituting the condition (4.4) into equation (3.5), we obtain the following recursive relations:

$$
y_{0}(t)=A t, \quad y_{i+1}(t)=y_{i}(t)-(\alpha-1) I^{\alpha}\left[t y_{i}{ }^{2}(t)-2-t^{5}\right], \ldots
$$

hence, we have

$$
\begin{aligned}
& y_{0}(t)=A t \\
& y_{1}(t)=y_{0}(t)-(\alpha-1) I^{\alpha}\left[t y_{0}^{2}(t)-2-t^{5}\right], \\
& y_{2}(t)=y_{1}(t)-(\alpha-1) I^{\alpha}\left[t y_{1}^{2}(t)-2-t^{5}\right], \\
& y_{3}(t)=y_{2}(t)-(\alpha-1) I^{\alpha}\left[t y_{2}{ }^{2}(t)-2-t^{5}\right]
\end{aligned}
$$

By using the above recursive relationship, we have

$$
y_{0}(t)=A t, \quad y_{1}(t)=A t-(\alpha-1)\left(\frac{A^{2} \Gamma(4)}{\Gamma(f f+4)} t^{f f+3}-\frac{2 \Gamma(2)}{\Gamma(f f+2)} t^{f f+1}-\frac{\Gamma(6)}{\Gamma(\alpha+6)} t^{\alpha+5}\right),
$$

where $A$ can be determined by using the boundary condition $y(1)=1$ in $y_{1}(t)$ (see Table 2).

Table 2: Values of the constant A for different value of $\alpha$.
\begin{tabular}{|c|c|c|c|c|}
\hline & $\alpha=1.25$ & $\alpha=1.5$ & $\alpha=1.75$ & $\alpha=2$ \\
\hline A & 0.8055417187 & 0.6946948654 & 0.6560107423 & 0.6649692020 \\
\hline
\end{tabular}

The numerical results obtained for the equation (4.3) by using VIM are shown in Table 3. The evolution results of Example 4.2 when $\alpha=1.25,1.5,1.75$ and $\alpha=2$ comparing with the exact solution of the equation (4.3) by VIM are shown in Figure 1. The value $\alpha=2$ is the case which the approximate solution in a good agreement with the exact solution of the equation (4.3). 
Table 3: Numerical results of Example 4.2 by VIM.

\begin{tabular}{|c|c|c|c|c|c|}
\hline $\mathrm{T}$ & Exact & $\alpha=1.25$ & $\alpha=1.5$ & $\alpha=1.75$ & $\alpha=2$ \\
\hline 0.0 & 0.00000000 & 0.0000000000 & 0.0000000000 & 0.000000000 & 0.000000000 \\
\hline 0.1 & 0.01 & 0.08165558343 & 0.07042015485 & 0.06620372309 & 0.06683011129 \\
\hline 0.2 & 0.04 & 0.08165558343 & 0.1443027816 & 0.1352479772 & 0.1356538699 \\
\hline 0.3 & 0.09 & 0.2545749067 & 0.2231314766 & 0.2091037529 & 0.2084423923 \\
\hline 0.4 & 0.16 & 0.3466954330 & 0.3079622212 & 0.2894407486 & 0.2871337341 \\
\hline 0.5 & 0.25 & 0.4428916531 & 0.3996715857 & 0.3777783860 & 0.3736463594 \\
\hline 0.6 & 0.36 & 0.5433818778 & 0.4991066925 & 0.4756030503 & 0.4699286118 \\
\hline 0.7 & 0.49 & 0.6485102935 & 0.6072445839 & 0.5845139631 & 0.5780561863 \\
\hline 0.8 & 0.64 & 0.7588785007 & 0.7253863006 & 0.7064186103 & 0.7003896018 \\
\hline 0.9 & 0.81 & 0.8755027965 & 0.8553971091 & 0.8437905894 & 0.8398036758 \\
\hline 1.0 & 1.0 & 0.9999999999 & 1.000000000 & 1.000000000 & 1.000001000 \\
\hline
\end{tabular}

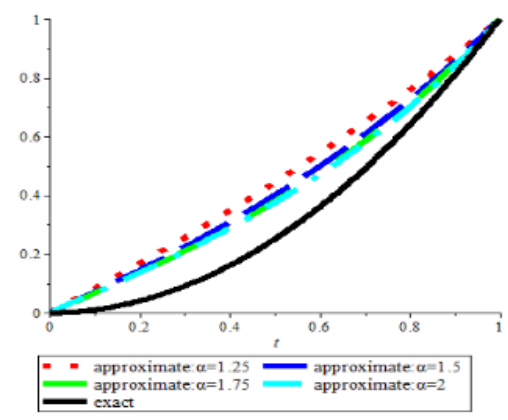

Figure 1: The evolution results of Example 4.2 when $\alpha=1.25,1.5,1.75$ and $\alpha=2$ comparing with the exact solution by VIM.

Example 4.3. Consider the following nonlinear fractional differential equation

$$
D_{*}^{\alpha} y(t)+e^{t} y^{3}(t)=e^{t}+e^{4 t}, \quad 1<\alpha \leqslant 2,
$$

subject to the boundary conditions

$$
y(0)=1, \quad y(1)=e,
$$

where the exact solution at $\alpha=2$ is:

$$
y(t)=e^{t} .
$$

The initial approximation $y_{0}(t)$ can be chosen in the form $y_{0}(t)=1+A t$. Substituting the initial condition (4.5) into equation (3.5), we obtain the following recursive relations:

$$
y_{0}(t)=1+A t, \quad y_{i+1}(t)=y_{i}(t)-(\alpha-1) I^{\alpha}\left[e^{t} y_{i}^{3}(t)-e^{t}-e^{4 t}\right], \ldots
$$

hence, we have

$$
\begin{aligned}
& y_{0}(t)=1+A t, \\
& y_{1}(t)=y_{0}(t)-(\alpha-1) I^{\alpha}\left[e^{t} y_{0}^{3}(t)-e^{t}-e^{4 t}\right], \\
& y_{2}(t)=y_{1}(t)-(\alpha-1) I^{\alpha}\left[e^{t} y_{1}^{3}(t)-e^{t}-e^{4 t}\right], \\
& y_{3}(t)=y_{2}(t)-(\alpha-1) I^{\alpha}\left[e^{t} y_{2}^{3}(t)-e^{t}-e^{4 t}\right] .
\end{aligned}
$$

In order to avoid the difficult fractional integration and to show the efficiency of this method, we taking the truncated Taylor expansion for the exponential terms in (4.6) by use the form $e^{t} \approx 1+t+\frac{t^{2}}{2}$, we have $y_{0}(t)=1+A t$, 


$$
\begin{aligned}
\mathrm{y}_{1}(\mathrm{t})= & 1+\mathrm{A} \mathrm{t}-(\alpha-1)\left(-\frac{\Gamma(1)}{\Gamma(\alpha+1)} \mathrm{t}^{\alpha}+\frac{3 \mathrm{~A} \Gamma(2)}{\Gamma(\alpha+2)} \mathrm{t}^{\alpha+1}+\frac{3 \mathrm{~A}^{2} \Gamma(3)}{\Gamma(\alpha+3)} \mathrm{t}^{\alpha+2}+\frac{\mathrm{A}^{3} \Gamma(4)}{\Gamma(\alpha+4)} \mathrm{t}^{\alpha+3}\right. \\
& -\frac{4 \Gamma(2)}{\Gamma(\alpha+2)} \mathrm{t}^{\alpha+1}+\frac{3 \mathrm{~A} \Gamma(3)}{\Gamma(\alpha+3)} \mathrm{t}^{\alpha+2}+\frac{3 \mathrm{~A}^{2} \Gamma(4)}{\Gamma(\alpha+4)} \mathrm{t}^{\alpha+3}+\frac{\mathrm{A}^{3} \Gamma(5)}{\Gamma(\alpha+5)} \mathrm{t}^{\alpha+4}-\frac{8 \Gamma(3)}{\Gamma(\alpha+3)} \mathrm{t}^{\alpha+2}+\frac{(1.5) \mathrm{A} \Gamma(4)}{\Gamma(\alpha+4)} \mathrm{t}^{\alpha+3} \\
& +\frac{(1.5) \mathrm{A}^{2} \Gamma(5)}{\Gamma(\alpha+5)} \mathrm{t}^{\alpha+4}+\frac{(0.5) \mathrm{A}^{3} \Gamma(6)}{\Gamma(\alpha+6)} \mathrm{t}^{\alpha+5}-\frac{(10) \Gamma(4)}{\Gamma(\alpha+4)} \mathrm{t}^{\alpha+3}-\frac{(8.5) \Gamma(5)}{\Gamma(\alpha+5)} \mathrm{t}^{\alpha+4}-\frac{5 \Gamma(6)}{\Gamma(\alpha+6)} \mathrm{t}^{\alpha+5} \\
& \left.-\frac{2 \Gamma(7)}{\Gamma(\alpha+7)} \mathrm{t}^{\alpha+6}-\frac{(0.5) \Gamma(8)}{\Gamma(\alpha+8)} \mathrm{t}^{\alpha+7}-\frac{\left(\frac{1}{16}\right) \Gamma(9)}{\Gamma(\alpha+9)} \mathrm{t}^{\alpha+8}\right),
\end{aligned}
$$

where $A$ can be determined by using the boundary condition $y(1)=e$ in $y_{1}(t)$ (see Table 4 ).

Table 4: Values of the constant $A$ for different value of $\alpha$.

\begin{tabular}{|c|c|c|c|c|}
\hline & $\alpha=1.25$ & $\alpha=1.5$ & $\alpha=1.75$ & $\alpha=2$ \\
\hline$A$ & 1.366180567 & 1.454677539 & 1.479241881 & 1.494118634 \\
\hline
\end{tabular}

The numerical results obtained for the equation (4.6) by using VIM are shown in Table 5. The evolution results of Example 4.3, when $\alpha=1.25,1.5,1.75$ and $\alpha=2$ comparing with the exact solution of the equation 4.17 by VIM are shown in Figure 2. The value $\alpha=1.25$ is the case which the approximate solution in a good agreement with the exact solution of the equation (4.6).

Table 5: Numerical results of Example 4.3 by VIM.

\begin{tabular}{|c|c|c|c|c|c|}
\hline $\mathrm{t}$ & Exact & $\alpha=1.25$ & $\alpha=1.5$ & $\alpha=1.75$ & $\alpha=2$ \\
\hline 0.0 & 1.00000000 & 1.0000000000 & 1.0000000000 & 1.000000000 & 1.000000000 \\
\hline 0.1 & 1.105170918 & 1.148914452 & 1.157112401 & 1.156034381 & 1.154303906 \\
\hline 0.2 & 1.221402758 & 1.301958627 & 1.322714211 & 1.322157314 & 1.317725253 \\
\hline 0.3 & 1.349858808 & 1.456346111 & 1.491825441 & 1.494532945 & 1.488706504 \\
\hline 0.4 & 1.491824698 & 1.611255242 & 1.661502909 & 1.669966292 & 1.664958372 \\
\hline 0.5 & 1.648721271 & 1.766888474 & 1.829563376 & 1.845376458 & 1.843616780 \\
\hline 0.6 & 1.822118800 & 1.924893765 & 1.994984895 & 2.018212650 & 2.021646147 \\
\hline 0.7 & 2.013752707 & 2.089152825 & 2.158846946 & 2.187357719 & 2.196597597 \\
\hline 0.8 & 2.225540928 & 2.266910201 & 2.325785238 & 2.354576006 & 2.367857312 \\
\hline 0.9 & 2.459603111 & 2.470293628 & 2.506050272 & 2.526638686 & 2.538550418 \\
\hline 1.0 & 2.718281828 & 2.718300001 & 2.718300000 & 2.718300000 & 2.718300001 \\
\hline
\end{tabular}

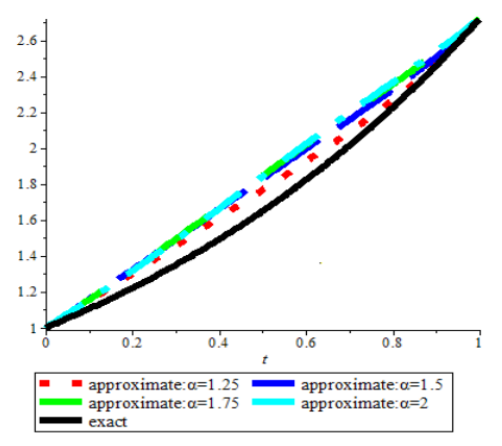

Figure 2: The evolution results of Example 4.3, when $\alpha=1.25,1.5,1.75$, and $\alpha=2$ comparing with the exact solution by VIM.

Example 4.4. Consider the following nonlinear fractional differential equation

$$
D_{*}^{\alpha} y(t)+t y^{2}(t)=-\sin t+t \sin ^{2} t, \quad 1<\alpha \leqslant 2,
$$


subject to the boundary conditions

$$
y(0)=0, \quad y\left(\frac{\pi}{2}\right)=1
$$

where the exact solution at $\alpha=2$ is:

$$
y(t)=\sin t
$$

By substitute the initial condition (4.8) into equation (3.5), we obtain the following recursive relations:

$$
y_{0}(t)=A t, \quad y_{i+1}(t)=y_{i}(t)-(\alpha-1) I^{\alpha}\left[t y_{i}^{2}(t)+\sin t-t \sin ^{2}\right]
$$

hence, we have

$$
\begin{aligned}
& y_{0}(t)=A t, \\
& y_{1}(t)=y_{0}(t)-(\alpha-1) I^{\alpha}\left[t y_{0}^{2}(t)+\sin t-t \sin ^{2} t\right], \\
& y_{2}(t)=y_{1}(t)-(\alpha-1) I^{\alpha}\left[t y_{1}{ }^{2}(t)+\sin t-t \sin ^{2} t\right], \\
& y_{3}(t)=y_{2}(t)-(\alpha-1) I^{\alpha}\left[t y_{2}{ }^{2}(t)+\sin t-t \sin ^{2} t\right] .
\end{aligned}
$$

In order to avoid the difficult fractional integration and to show the efficiency of this method, we take the truncated Taylor expansion for the function $\sin t$ in (4.9) by the form $\sin t=t-\frac{t^{3}}{6}+\frac{t^{5}}{120}$, hence we have

$$
\begin{aligned}
y_{0}(t)= & A t \\
y_{1}(t)= & A t-(\alpha-1)\left(\frac{\Gamma(2)}{\Gamma(\alpha+2)} t^{\alpha+1}+\frac{A^{2} \Gamma(4)}{\Gamma(\alpha+4)} t^{\alpha+3}-\frac{\left(\frac{7}{6}\right) \Gamma(4)}{\Gamma(\alpha+4)} t^{\alpha+3}+\frac{\left(\frac{41}{120}\right) \Gamma(6)}{\Gamma(\alpha+6)} t^{\alpha+5}\right. \\
& -\frac{\left(\frac{2}{45}\right) \Gamma(8)}{\Gamma(\alpha+8)} t^{\alpha+7}+\frac{\left(\frac{1}{360}\right) \Gamma(10)}{\Gamma(\alpha+10)} t^{\alpha+9}-\frac{\left(\frac{1}{14400}\right) \Gamma(12)}{\Gamma(\alpha+12)} t^{\alpha+11),}
\end{aligned}
$$

where $A$ can be determined by using the boundary condition $y\left(\frac{\pi}{2}\right)=1$ in $y_{1}(t)$ (see Table 6). The

Table 6: Values of the constant $A$ for different values of $\alpha$.

\begin{tabular}{|c|c|c|c|c|}
\hline & $\alpha=1.25$ & $\alpha=1.5$ & $\alpha=1.75$ & $\alpha=2$ \\
\hline$A$ & 0.7823792232 & 0.9842587260 & 1.226212972 & 1.347858413 \\
\hline
\end{tabular}

numerical results obtained for the equation (4.7) by using VIM are shown in Table 7. The evolution results of Example 4.4, when $\alpha=1.25,1.5,1.75$, and $\alpha=2$ comparing with the exact solution of the equation (4.7) by VIM are shown in Figure 3. The value $\alpha=1.5$ is the case which the approximate solution in a good agreement with the exact solution of (4.7).

Table 7: Numerical results of Example 4.4 by VIM.

\begin{tabular}{|c|c|c|c|c|c|}
\hline $\mathrm{t}$ & Exact & $\alpha=1.25$ & $\alpha=1.5$ & $\alpha=1.75$ & $\alpha=2$ \\
\hline 0.0 & 0.00000000 & 0.0000000000 & 0.0000000000 & 0.000000000 & 0.000000000 \\
\hline$\frac{\pi}{16}$ & 0.1950907723 & 0.1511264131 & 0.1906961696 & 0.2388299842 & 0.2633807649 \\
\hline$\frac{\pi}{8}$ & 0.3826842808 & 0.2956877538 & 0.3721232083 & 0.4683165450 & 0.5188956128 \\
\hline$\frac{3 \pi}{16}$ & 0.5555713783 & 0.4332271426 & 0.5404188940 & 0.6809074862 & 0.7573912083 \\
\hline$\frac{\pi}{4}$ & 0.7071080799 & 0.5641414795 & 0.6924528671 & 0.8678183155 & 0.9667172289 \\
\hline$\frac{5 \pi}{16}$ & 0.8314708878 & 0.6886169428 & 0.8241059215 & 1.016789294 & 1.129261448 \\
\hline$\frac{3 \pi}{8}$ & 0.9238805868 & 0.8055489413 & 0.9283840258 & 1.109348700 & 1.218558349 \\
\hline$\frac{7 \pi}{16}$ & 0.9807859074 & 0.9114824045 & 0.9933407797 & 1.117568393 & 1.195005526 \\
\hline$\frac{\pi}{2}$ & 1.000000000 & 0.9997668569 & 1.000094232 & 1.000603048 & 1.000930472 \\
\hline
\end{tabular}




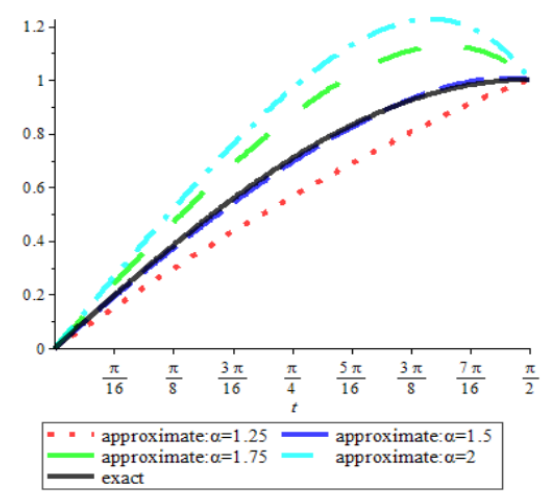

Figure 3: The evolution results of Example 4.4, when $\alpha=1.25,1.5,1.75$, and $\alpha=2$ comparing with the exact solution by VIM.

\section{Conclusion}

In this paper variational iteration method has been successfully applied to find the solution of a non linear fractional differential equations are presented in Tables 3, 5, and 7 for differential results of $t$ to show the stability of the method. The approximate solutions obtained by variational iteration method are compared with the exact solutions which shown in Figures 1, 2, and 3.

\section{References}

[1] A. Arikoglu, I. Ozkol, Solution of fractional integro-differential equations by using fractional differential transform method, Chaos Solitons Fractals, 40 (2009), 521-529. 1

[2] Z. D. Cen, A. B. Le, A. M. Xu, A robust numerical method for a fractional differential equation, Appl. Math. Comput., 315 (2017) 654-671. 1

[3] Y. M. Chen, M. X. Yi, C. X. Yu, Error analysis for numerical solution of functional differential equation by Haar wavelets method, J. Comput. Sci., 5 (2012), 367-573. 1

[4] K. Diethelm, D. Baleanu, E. Scalas, J. J. Trujillo, Fractional calculus: models and numerical methods, World Scientific Publishing Co., Hackensack, (2012). 2.1, 2.3

[5] I. L. El-Kalla, Error estimate of the series solution to a class of nonlinear fractional differential equations, Commun. Nonlinear Sci. Numer. Simul., 16 (2011), 1408-1413. 1

[6] A. M. A. El-Sayed, Nonlinear functional differential equations of arbitrary orders, Nonlinear Anal., 33 (1998), $181-186$. 1

[7] L.-L. Huang, B.-Q. Liu, D. Baleanu, G.-C. Wu, Numerical solutions of interval-valued fractional nonlinear differential equations, Eur. Phys. J. PLUS, 134 (2019), 15 pages.

[8] H. Jafari, A. Kadem, D. Baleanu, T. Yilmaz, Solutions of the fractional Davey-Stewartson equations with variational iteration method, Romanian Reports in Physics, 64 (2012), 337-346. 1, 3

[9] H. Jafari, H. Tajadodi, D. Baleanu, A numerical a approach for fractional order Riccati differential equation using B-Spline operational matrix, Fract. Calc. Appl. Anal., 18 (2015), 387-399. 1

[10] A. Kadem, A. Kilicman, The approximate solution of fractional Fredholm integrodifferential equations by variational iteration and homotopy perturbation methods, Abstr. Appl. Anal., 2012 (2012), 10 pages. 1, 3

[11] W. J. Li, Y. Peng, Application of Adomian decomposition method to nonlinear system, Adv. Difference Equ., 2020 (2020), 67 pages. 1

[12] A. Loverro, Fractional Calculus: History, Definitions and Applications for the Engineer, Rapport technique (Univeristy of Notre Dame, Department of Aerospace and Mechanical Engineering), 2004 (2004), 28 pages. 2

[13] E. M. A. M. Mendes, G. H. O. Salgado, L. A. Aguirre, Numerical solution of Caputo fractional differential equations with infinity memory effect at initial condition, Commun. Nonlinear Sci. Numer. Simul., 69 (2019), 237-247.

[14] Z. J. Meng, M. X. Yi, J. Huang, L. Song, Numerical solutions of nonlinear fractional differential equations by alternative Legendre polynomials, Appl. Math. Comput., 336 (2018), 454-464. 1

[15] S. Momani, M. Aslam Noor, Numerical methods for fourth order fractional integro-differential equations, Appl. Math. Comput., 182 (2006), 754-760. 1

[16] S. Momani, R. Qaralleh, An efficient method for solving systems of fractional Integro-differential equations, Comput. Math. Appl., 52 (2006), 459-470. 1

[17] Y. Nawaz, Variational iteration method and homotopy perturbation method for fourth-order fractional integro-differential equations, Comput. Math. Appl., 61 (2011), 2330-2341. 1, 3 
[18] I. Podlubny, Fractional differential equations, Academic press, San Diego, (1999). 2.2, 2.4

[19] E. A. Rawashdeh, Numerical solution of fractional integro-differential equations by collocation method, Appl. Math. Comput., 176 (2006), 1-6. 1

[20] S. S. Ray, Numerical Solutions of Riesz Fractional Partial Differential Equations, Nonlinear Diff. Equ. Phys., 2020 (2020), 119-154.

[21] A. Saadatmandi, M. Dehghan, A new operational matrix for solving fractional-order differential equations, Comput. Math. Appl., 59 (2010), 1326-1336. 1

[22] J. Saberi-Nadjafi, M. Tamamgar, The variational iteration method: A highly promising method for solving the system of integro-differential equations, Comput. Math. Appl., 56 (2008), 346-351. 1, 3

[23] M. R. A. Sakran, Numerical Solutions integral and integro-differential equations using Chebyshev polynomials of the third kind, Appl. Math. Comput., 351 (2019), 66-82.

[24] M. S. Semary, H. N. Hassan, A. G. Radwan, Modified methods for solving two classes of distributed order linear fractional differential equations, Appl. Math. Comput., 323 (2018), 106-119.

[25] D. Yang, J. R. Wang, D. O'Regan, A class of nonlinear non-instantaneous impulsive differential equations involving parameters and fractional order, Appl. Math. Comput., 321 (2018), 654-671. 1

[26] M. X. Yi , Y. M. Chen, Haar wavelet operational matrix method for solving fractional partial differential equations, CMES Comput. Model. Eng. Sci., 88 (2012), 229-244. 1 